\title{
Harboring Cnm-Expressing Streptococcus Mutans In The Oral Cavity Relates To Both Deep and Lobar Cerebral Microbleeds
}

Shuhei Ikeda

National Cerebral and Cardiovascular Center

Satoshi Saito

National Cerebral and Cardiovascular Center

Satoshi Hosoki

National Cerebral and Cardiovascular Center

Shuichi Tonomura

National Cerebral and Cardiovascular Center

Yumi Yamamoto

National Cerebral and Cardiovascular Center

Hajime Ikenouchi

National Cerebral and Cardiovascular Center

Hiroyuki Ishiyama

National Cerebral and Cardiovascular Center

Tomotaka Tanaka

National Cerebral and Cardiovascular Center

Yorito Hattori

National Cerebral and Cardiovascular Center

Robert P. Friedland

University of Louisville

Roxana 0. Carare

University of Southampton

Nagato Kuriyama

Shizuoka Graduate University of Public Health

Yusuke Yakushiji

Saga University Faculty of Medicine

Hideo Hara

Saga University Faculty of Medicine

Masatoshi Koga

National Cerebral and Cardiovascular Center

Kazunori Toyoda 
National Cerebral and Cardiovascular Center

\section{Ryota Nomura}

Osaka University Graduate School of Dentistry

\section{Misa Takegami}

National Cerebral and Cardiovascular Center

\section{Kazuhiko Nakano}

Osaka University Graduate School of Dentistry

\section{Masafumi lhara ( $D$ ihara@ncvc.go.jp)}

National Cerebral and Cardiovascular Center

\section{Research Article}

Keywords: Streptococcus, cariogenic bacterium, cerebrovascular, microbleeds

Posted Date: December 28th, 2021

DOI: https://doi.org/10.21203/rs.3.rs-1193993/v1

License: (c) (i) This work is licensed under a Creative Commons Attribution 4.0 International License. Read Full License 


\section{Abstract}

Streptococcus mutans, a major cariogenic bacterium, expressing the collagen-binding protein $\mathrm{Cnm}$ induces cerebrovascular inflammation, resulting in the impairment of blood brain barrier integrity followed by cerebral bleeding. We here examined the association of $\mathrm{Cnm}$-positive S. mutans with cerebral microbleeds (CMBs) in acute stroke patients selected from a single-center registry database. Of 428 patients who received oral bacterial examinations among 3154 stroke patients, 326 patients who harbored $S$. mutans were identified. After excluding four patients without imaging data, we compared 72 patients with $\mathrm{Cnm}$-positive S. mutans and 250 with Cnm-negative S. mutans. Deep, lobar and infratentorial CMBs were observed in 46 (63.9\%), 36 (50.0\%), 25 (34.7\%) patients with Cnm-positive $S$. mutans and 144 (57.6\%), 114 (45.6\%), 101 (40.4\%) with Cnm-negative S. mutans. Possession of Cnmpositive $S$. mutans was related to higher numbers of both deep and lobar, but not infratentorial, CMBs (risk ratios 1.57 [1.07-2.30], deep; 5.44 [2.50-11.85], lobar). Statistical significance persisted after adjusting for age, sex, hypertension, stroke type, National Institutes of Health Stroke Scale score, and cerebral amyloid angiopathy (risk ratios 1.61 [1.14-2.27], deep; 5.14 [2.78-9.51], lobar). Our study indicated that reduction of $\mathrm{Cnm}$-positive $S$. mutans may serve as a therapeutic approach for improving the prognosis of stroke patients.

\section{Introduction}

Cerebral microbleeds (CMBs), an independent predictor of dementia and stroke $\mathrm{e}^{1-3}$, are radiologically defined constructs found on magnetic resonance imaging $(\mathrm{MRI})^{4,5}$. The underlying pathology of most CMBs consists of iron-positive focal or dispersed accumulations of siderophages corresponding to previous hemorrhages from small cerebral vessels ${ }^{6,7}$. However, CMBs are also accompanied by various pathological lesions such as inflammatory vasculitis and fibrinoid necrosis ${ }^{6}$; therefore, clarifying the pathogenesis of CMBs remains challenging ${ }^{4}$.

Hypertensive arteriopathy and cerebral amyloid angiopathy (CAA) are the two most common causes of CMBs. Previous studies have suggested that deep CMBs are mostly associated with hypertensive arteriopathy, whereas lobar $\mathrm{CMBs}$ reflect $\mathrm{CA} \mathrm{A}^{8}$. However, growing evidence has shown that hypertensive arteriopathy induces both deep and lobar $\mathrm{CMBs}^{8}$. One pathological investigation showed that lobar CMBs were related to hypertensive arteriopathy in the absence of $\mathrm{CAA}^{7}$.

We previously reported that oral carriage of Streptococcus mutans, a major cariogenic bacterium, expressing the $\mathrm{Cnm}$ protein is related to an increased risk of deep $\mathrm{CMBs}^{9-11}$. Cnm, encoded by the $\mathrm{cnm}$ gene, is a cell-surface $120-\mathrm{kD}$ a collagen-binding protein of $S$. mutans ${ }^{12}$. Intravenous administration of Cnm-expressing S. mutans (Cnm-positive S. mutans) aggravates cerebral bleeding in both the cortical and deep gray matter in stroke-prone spontaneously hypertensive rats (SHRs) ${ }^{13}$. Nevertheless, the contribution of $\mathrm{Cnm}$-positive $S$. mutans to lobar CMBs in humans remains unclear ${ }^{9,14,15}$. Infective endocarditis is a critical consequence of dental bacteremia including that caused by $\mathrm{Cnm}$-positive $S$. 
mutans $^{16}$, and lobar CMBs precede intracerebral hemorrhage $(\mathrm{ICH})$ in infective endocarditis ${ }^{17}$. We therefore hypothesized that $\mathrm{Cnm}$-positive $S$. mutans is associated with the development of lobar and deep CMBs. This cross-sectional study investigated the involvement of $\mathrm{Cnm}$-positive $\mathrm{S}$. mutans in deep and lobar CMBs in stroke patients.

\section{Results}

\section{Patient Selection}

Among 3154 stroke patients, 428 patients (13.6\%) underwent an oral bacterial examination (Figure 1). The characteristics of these 428 patients are described in the Supplementary Materials (see Supplementary Table 1). Patients who underwent oral bacterial examinations had younger ages and lower National Institutes of Health Stroke Scale (NIHSS) scores compared to those who did not undergo examinations (age: 73.0 [63.0-81.0] years versus 76.0 [67.0-83.0] years, $P<0.001$; NIHSS score: 3.0 [1.0-6.0] versus 4.0 [1.0-14.0], $P<0.001)$. In addition, higher frequencies of $\mathrm{ICH}$ and hypertension and a lower prevalence of atrial fibrillation were observed in patients who underwent a bacterial examination (ICH: $36.9 \%$ versus $20.1 \%, P<0.001$; hypertension: $89.3 \%$ versus $82.1 \%, P<0.001$ : atrial fibrillation: $17.3 \%$ versus $24.0 \%, P=0.002)$.

In the oral bacterial examination, S. mutans was detected in 326 patients $(76.2 \%)$ and was absent in the remaining 102 patients (23.8\%). The clinical profiles were similar between patients with and without $S$. mutans in the oral cavity (see Supplementary Table 2). We identified 72 patients with the Cnm-positive $S$. mutans (Cnm [+] group) and 254 with the Cnm-negative S. mutans (Cnm [-] group). Four patients in the Cnm (-) group were excluded from the analyses because no MRI data were available.

\section{Demographics and Clinical Characteristics}

The clinical profiles of the $\mathrm{Cnm}(+)$ and $\mathrm{Cnm}(-)$ groups are described in Table 1. The age, sex, blood pressure, vascular risk factors, and medication histories were similar between the two groups. The $\mathrm{Cnm}$ $(+)$ group showed slightly lower NIHSS scores than the Cnm (-) group (2.0 [1.0-5.0] versus 3.0 [1.0-7.3], $P=0.125)$. The prevalence of CAA was comparable between the two groups. 
Table 1

Clinical profiles of Cnm-positive and negative groups

\begin{tabular}{|c|c|c|c|}
\hline & $\begin{array}{l}\text { Cnm (+) group } \\
(n=72)\end{array}$ & $\begin{array}{l}\text { Cnm }(-) \text { group } \\
(n=250)\end{array}$ & $P$ value \\
\hline Age, y & $72.5(61.0-79.0)$ & $72.0(62.0-81.0)$ & 0.554 \\
\hline Males, n (\%) & $48(66.7)$ & $161(64.4)$ & 0.723 \\
\hline Systolic blood pressure, $\mathrm{mmHg}$ & $124.0(117.3-134.0)$ & $120.0(113.0-132.3)$ & 0.394 \\
\hline Diastolic blood pressure, $\mathrm{mmHg}$ & $72.0(65.0-79.8)$ & $70.0(61.0-78.3)$ & 0.338 \\
\hline \multicolumn{4}{|l|}{ Vascular risk factors } \\
\hline Hypertension, n (\%) & $67(93.1)$ & $222(88.8)$ & 0.294 \\
\hline Dyslipidemia, n (\%) & $41(56.9)$ & $122(48.8)$ & 0.223 \\
\hline Diabetes, n (\%) & $19(26.4)$ & $51(20.4)$ & 0.278 \\
\hline Atrial fibrillation, $\mathrm{n}(\%)$ & $11(15.3)$ & $43(17.2)$ & 0.700 \\
\hline Smoking, n (\%) & $14(19.4)$ & $46(18.4)$ & 0.841 \\
\hline \multicolumn{4}{|l|}{ Medication } \\
\hline Antihypertensive agents, n (\%) & $40(55.6)$ & $140(56.0)$ & 0.947 \\
\hline ATAs, $n(\%)$ & $31(43.1)$ & $97(38.8)$ & 0.516 \\
\hline Antiplatelet agents, n (\%) & $20(27.8)$ & $71(28.4)$ & 0.918 \\
\hline Anticoagulants, n (\%) & $13(18.1)$ & $35(14.0)$ & 0.395 \\
\hline Use of $\geq 2$ ATAs, $n(\%)$ & $4(5.6)$ & $15(6.0)$ & 1.000 \\
\hline CAA & & & 0.316 \\
\hline Probable CAA, n (\%) & $6(8.3)$ & $10(4.0)$ & \\
\hline Possible CAA, n (\%) & $1(1.4)$ & $7(2.8)$ & \\
\hline No CAA, n (\%) & $65(90.3)$ & $233(93.2)$ & \\
\hline CMBs, n (\%) & & & 0.141 \\
\hline Strictly lobar CMBs, n (\%) & $5(6.9)$ & $8(3.2)$ & \\
\hline $\mathrm{D} / \mathrm{l}$ or mixed $\mathrm{CMBs}, \mathrm{n}(\%)$ & $50(69.4)$ & $158(63.2)$ & \\
\hline
\end{tabular}

Data represent the median (interquartile range) or number (percent). Cnm (+), Cnm-positive group; Cnm (-), Cnm-negative group; ATAs, anti-thrombotic agents; CAA, cerebral amyloid angiopathy; CMBs, cerebral microbleeds; D/I, deep and/or infratentorial; ICH, intracerebral hemorrhage; NIHSS, National Institutes of Health Stroke Scale. 


\begin{tabular}{|c|c|c|c|}
\hline & $\begin{array}{l}\text { Cnm (+) group } \\
(n=72)\end{array}$ & $\begin{array}{l}\text { Cnm }(-) \text { group } \\
(n=250)\end{array}$ & $P$ value \\
\hline No CMBs, n (\%) & $17(23.6)$ & $84(33.6)$ & \\
\hline Stroke type & & & 0.843 \\
\hline Ischemic stroke, n (\%) & $44(61.1)$ & $156(62.4)$ & \\
\hline $\mathrm{ICH}, \mathrm{n}(\%)$ & $28(38.9)$ & $94(37.6)$ & \\
\hline NIHSS score & $2.0(1.0-5.0)$ & $3.0(1.0-7.3)$ & 0.125 \\
\hline \multicolumn{4}{|c|}{$\begin{array}{l}\text { Data represent the median (interquartile range) or number (percent). Cnm (+), Cnm-positive group; } \\
\text { Cnm (-), Cnm-negative group; ATAs, anti-thrombotic agents; CAA, cerebral amyloid angiopathy; CMBs } \\
\text { cerebral microbleeds; D /I, deep and/or infratentorial; ICH, intracerebral hemorrhage; NIHSS, National } \\
\text { Institutes of Health Stroke Scale. }\end{array}$} \\
\hline
\end{tabular}

\section{Cerebral Microbleeds}

CMBs were observed in 55 (76.4\%) patients in the $\mathrm{Cnm}(+)$ group and $166(66.4 \%)$ in the Cnm (-) group $(P=0.107)$. Strictly lobar CMBs were found in 5 patients $(6.9 \%)$ in the $\mathrm{Cnm}(+)$ group and $8(3.2 \%)$ in the $\mathrm{Cnm}(-)$ group $(P=0.175)$. The median (interquartile range) numbers of $\mathrm{CMBs}$ in the $\mathrm{Cnm}(+)$ group were $4.0(1.0-11.0)$ for all CMBs, $1.0(0.0-6.5)$ for deep CMBs, $0.5(0.0-4.8)$ for lobar CMBs, and $0.0(0.0-1.0)$ for infratentorial CMBs, while the numbers of CMBs in the $\mathrm{Cnm}(-)$ group were $2.0(0.0-7.0)$ for all, 1.0 $(0.0-3.3)$ for deep, $0.0(0.0-2.0)$ for lobar, and $0.0(0.0-1.3)$ for infratentorial CMBs. When the number of CMBs was stratified into six categories, the $\mathrm{Cnm}(+)$ group was significantly distributed in the "higher CMB" categories for all and lobar CMBs but not for deep or infratentorial CMBs (all CMBs, $P=0.030$; deep, $P=0.178$; lobar, $P=0.009$; infratentorial, $P=0.721$, Table 2). 
Table 2

Number of CMBs in the Cnm-positive and negative groups

Number of CMBs

$P$ value

\begin{tabular}{|c|c|c|c|c|c|c|c|}
\hline & 0 & 1 & $2-4$ & $5-10$ & $11-20$ & $\geq 21$ & \\
\hline \multicolumn{8}{|l|}{ All CMBs } \\
\hline Cnm (+) & $17(23.6)$ & $8(11.1)$ & $15(20.8)$ & $11(15.3)$ & $9(12.5)$ & $12(16.7)$ & 0.030 \\
\hline Cnm (-) & $84(33.6)$ & $25(10.0)$ & $46(18.4)$ & $53(21.2)$ & $27(10.8)$ & $15(6.0)$ & \\
\hline \multicolumn{8}{|c|}{ Deep CMBs } \\
\hline Cnm (+) & $26(36.1)$ & $13(18.1)$ & $13(18.1)$ & $13(18.1)$ & $5(6.9)$ & $2(2.8)$ & 0.178 \\
\hline Cnm (-) & $106(42.4)$ & 39 (15.6) & $55(22.0)$ & $40(16.0)$ & $6(2.4)$ & $4(1.6)$ & \\
\hline \multicolumn{8}{|c|}{ Lobar CMBs } \\
\hline Cnm (+) & $36(50.0)$ & $6(8.3)$ & $12(16.7)$ & $8(11.1)$ & $4(5.6)$ & $6(8.3)$ & 0.009 \\
\hline Cnm (-) & $136(54.4)$ & $40(16.0)$ & $43(17.2)$ & $25(10.0)$ & $2(0.8)$ & $4(1.6)$ & \\
\hline \multicolumn{8}{|c|}{ Infratentorial CMBs } \\
\hline Cnm (+) & $47(65.3)$ & $10(13.9)$ & $8(11.1)$ & $5(6.9)$ & $0(0)$ & $2(2.8)$ & 0.721 \\
\hline Cnm (-) & $149(59.6)$ & 39 (15.6) & $38(15.2)$ & $21(8.4)$ & $1(0.4)$ & $2(0.8)$ & \\
\hline
\end{tabular}

Table 3 shows the unadjusted and adjusted risk ratios between the presence of Cnm-positive $S$. mutans and the number of CMBs. Harboring Cnm-positive $S$. mutans was significantly associated with a greater number of all, deep, and lobar CMBs, but not infratentorial CMBs, in the unadjusted model (risk ratio of all CMBs, 2.84 [95\% confidence interval, 1.66-4.87], $P<0.001$; deep CMBs, 1.57 [1.07-2.30], $P=0.021$; lobar CMBs, 5.44 [2.50-11.85], $P<0.001$; infratentorial CMBs, 1.55 [0.72-3.33], $P<0.263)$. Statistical significance persisted after adjusting for age, sex, hypertension, stroke type, NIHSS score, and CAA (adjusted risk ratio of all CMBs, 2.73 [1.72-4.33], $P<0.001$; deep CMBs, 1.61 [1.14-2.27], $P<0.001$; lobar CMBs, 5.14 [2.78-9.51], $P<0.001)$. 
Table 3

Risk ratios and $95 \%$ confidence intervals for associations between $\mathrm{Cnm}$-positive $S$. mutans and the number of CMBs

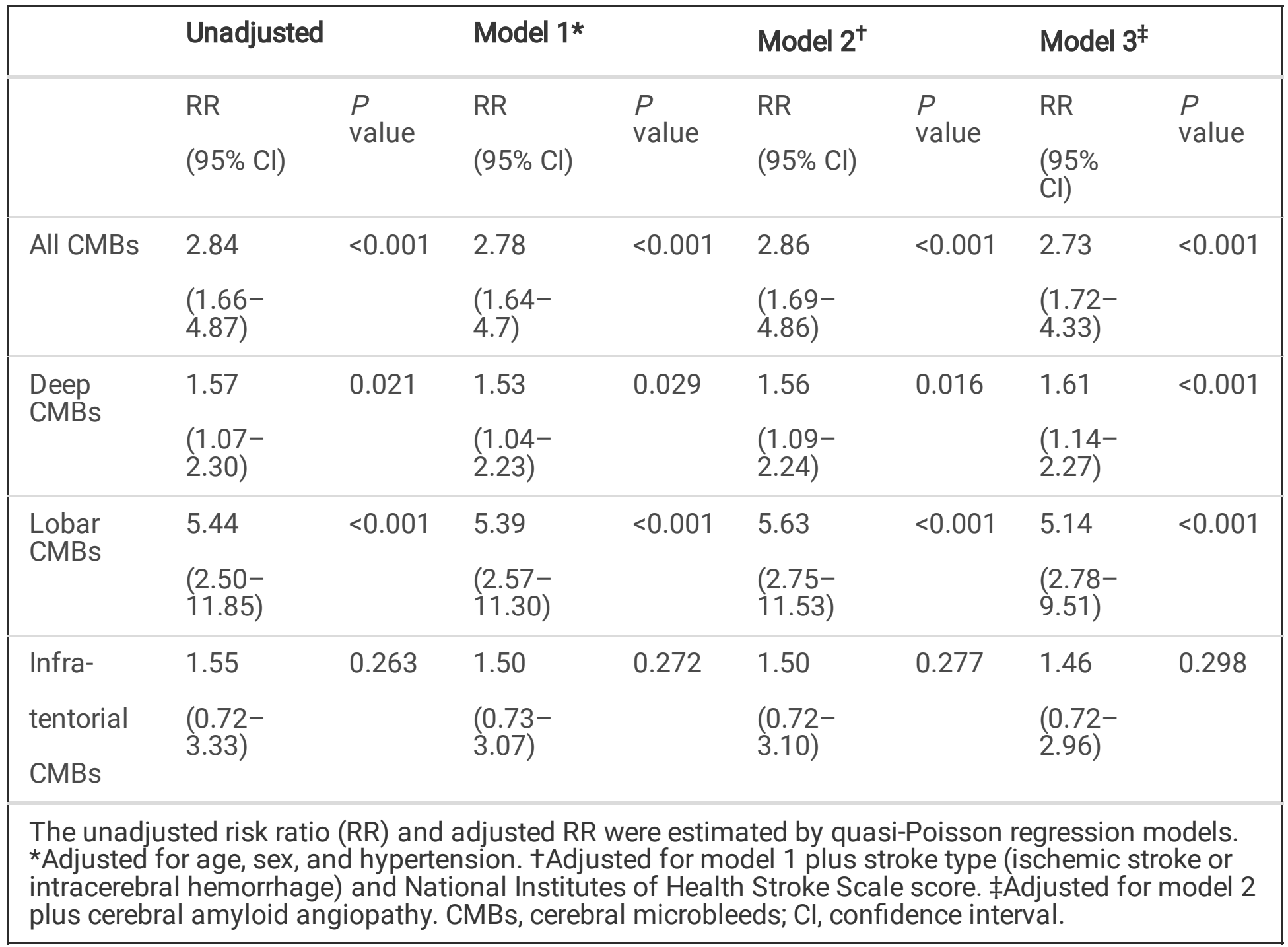

The inter-rater correlation coefficients for deep, lobar, and infratentorial CMBs were $0.87,0.88$, and 0.95 , respectively. Representative images showing a patient with a substantial number of deep and lobar CMBs are illustrated in Figure 2.

\section{Discussion}

The current study demonstrated that oral carriage of Cnm-positive $S$. mutans was independently associated with a greater number of all, deep, and lobar, but not infratentorial, CMBs. The similar frequencies of CAA and strictly lobar CMBs between patients with Cnm-positive and those with Cnmnegative $S$. mutans suggests that $\mathrm{Cnm}$-positive $S$. mutans does not accelerate the pathophysiology of CAA.

S. mutans is an anaerobic Gram-positive coccus that is detected in the oral cavity of approximately $90 \%$ of the general population ${ }^{15}$. Bacteremia caused by $S$. mutans is almost inevitable in daily life because of toothbrushing, flossing, or tooth extraction ${ }^{10}$. The major sources of $S$. mutans are mothers or 
caregivers $^{18}$. S. mutans is vertically transmitted and colonizes the mouths of infants ${ }^{18}$ and is rarely implanted during adulthood ${ }^{19}$. However, it frequently disappears from the oral cavity of edentulous people because $S$. mutans resides on the tooth surface ${ }^{20}$. We therefore excluded patients from whom no S. mutans was detected and compared those with $\mathrm{Cnm}$-positive and $\mathrm{Cnm}$-negative $S$. mutans in this study.

Cnm-positive $S$. mutans is characterized by its binding to components of the vascular basement membrane, such as collagen-IV and laminin ${ }^{12,16,21}$, while $\mathrm{Cnm}$-negative $S$. mutans cannot attach to soft tissues ${ }^{16,21}$. Aging and vascular risk factors including hypertension induce endothelial injury and increase the thickness of the basement membranes, resulting in collagen-IV and laminin exposure in small cerebral arteries ${ }^{22,23}$. Once Cnm-positive $S$. mutans adheres to the basement membrane ${ }^{21}$, infiltration of neutrophils may aggravate local inflammation, resulting in increased permeability of the blood-brain barrier and increased production of enzymes, such as matrix metalloproteinase- $9^{13}$, that accelerate endothelial damage, leading to CMBs (Figure 3$)^{11}$.

Our previous cross-sectional ${ }^{9}$ and retrospective longitudinal studies ${ }^{11}$ showed a strong association of Cnm-positive $S$. mutans with deep, but not lobar, CMBs. The seemingly different results regarding lobar CMBs may stem from the different sample sizes between the previous and current studies. Twenty-one or fewer stroke patients with $\mathrm{Cnm}$-positive $S$. mutans were registered in the previous studies ${ }^{9,11}$. Hachinski described the "vascular centrencephalon" as the phylogenetically ancient part of the brain that is perfused by short straight arteries with few branches, transmitting pressure directly from the large arteries to small arterioles ${ }^{24,25}$. However, the cortex is supplied by long arteries with many branches, resulting in a large blood pressure decrement in the brain ${ }^{26}$. In a computational hemodynamics model of hypertension patients with a blood pressure of $192 / 113 \mathrm{mmHg}$ in the brachial artery, the small arterial pressure was $169 / 101 \mathrm{mmHg}$ in the lenticulostriate bed but only $117 / 68 \mathrm{mmHg}$ in the posterior parietal artery bed ${ }^{26}$. This marked difference in the arterial pressure between the deep and cortical regions could explain why lacunar infarcts related to vasculopathies induced by high blood pressure preferentially occur in the vascular centrencephalon rather than in the cortex ${ }^{25}$.

However, stroke-prone SHRs, a rat model of systemic hypertension, exhibit endothelial damage not only in the deep arteries but also in the cortical arteries ${ }^{23}$. Furthermore, cerebrovascular integrity is more severely damaged in stroke-prone SHRs than in SHRs, even though stroke-prone SHRs and SHRs show similar degrees of hypertension ${ }^{27}$. Hypertensive patients who have achieved target blood pressure levels still display autonomic dysfunction ${ }^{28}$ and a high residual cardiovascular risk ${ }^{29}$. These findings suggest that factors other than high blood pressure also contribute to endothelial injury in patients with systemic hypertension ${ }^{10}$, which may explain the increased numbers of lobar and deep CMBs in patients with Cnmpositive $S$. mutans. Furthermore, patients with a high-salt diet exhibit impaired endothelium-dependent vasodilation even without any blood pressure changes ${ }^{30}$. The age of onset of hypertension is associated with end organ damage, independent of the measured blood pressure ${ }^{31}$. Hence, several known and 
unknown factors other than hypertension may contribute to endothelial injury in small arteries, enabling Cnm-positive S. mutans to attach to the basement membranes and induce both deep and lobar CMBs.

There are some limitations to this study. First, this study was retrospectively performed, posing a potential risk of selection bias. Second, only 428 patients (13.6\%) of the total 3154 stroke patients underwent an oral bacterial evaluation. We attempted to widely recruit stroke patients, but older and severe stroke patients tended not to participate in the study, largely because of difficulties in explaining the research to those with impaired consciousness or various disabilities including dementia and advanced frailty. This resulted in a younger age and lower NIHSS scores in patients who underwent bacterial assessments. Third, the proportion of patients with $\mathrm{ICH}$ and the frequencies of patients with a history of hypertension or atrial fibrillation were different between those who did and did not undergo oral bacterial examination. We previously reported that $\mathrm{Cnm}$-positive $S$. mutans is more closely associated with hypertensive $\mathrm{ICH}$ than ischemic stroke ${ }^{9}$, which might have influenced participation in the research or affected the success rate of informed consent acquisition. Fourth, while cognitive function was not assessed in this study, we are now performing a prospective observational study to evaluate the effects of Cnm-positive $S$. mutans on cognition.

In conclusion, we found that $\mathrm{Cnm}$-positive $S$. mutans was associated with a higher number of both lobar and deep CMBs. The close association between $\mathrm{Cnm}$-positive $S$. mutans and CMBs suggests that reduction of $\mathrm{Cnm}$-positive $S$. mutans in the oral cavity may serve as a novel therapeutic approach for improving the long-term prognosis of stroke patients.

\section{Methods}

\section{Data Availability Statement}

Raw data were generated and stored at the National Cerebral and Cardiovascular Center. Derived data supporting the findings of this study are available from the corresponding authors on request.

\section{Study Design}

The significance of oral carriage of Cnm-positive S. mutans with regard to CMBs was evaluated in a cross-sectional study in accordance with the Declaration of Helsinki standards and the Japanese Ethical Guidelines for Medical and Health Research Involving Human Subjects after approval was obtained from the Ethical Committee of the National Cerebral and Cardiovascular Center (M23-073-8, M27-015-5).

Acute stroke patients were selected from the National Cerebral and Cardiovascular Center Stroke Registry database (https://www.clinicaltrials.gov; Unique identifier: NCT02251665) who fully satisfied the following criteria: (1) patients who underwent oral bacterial assessments from February 2014 to May 2016 or from May 2017 to October 2018; (2) patients who developed ischemic stroke or ICH during the above period; (3) patients older than 40 years old; and (4) patients or their legal representative provided written informed consent for the current study. We did not perform oral bacterial assessments for any 
patients between June 2016 and April 2017. The sample size and study period were determined based on feasibility. Patients without MRI data were excluded from the analyses. Patients in Cnm (+) group were compared with those in $\mathrm{Cnm}(-)$ group. We also compared acute stroke patients receiving oral bacterial examination to those who did not receive the examination in order to uncover potential sources of bias.

\section{Clinical Characteristics}

Clinical information, except for MRI findings, was obtained from the National Cerebral and Cardiovascular Center Stroke Registry database. Hypertension was defined as systolic blood pressure $\geq 140 \mathrm{mmHg}$, diastolic blood pressure $\geq 90 \mathrm{mmHg}$, or a history of antihypertensive medication use. Diabetes mellitus was considered present when a patient used antidiabetic drugs or insulin, the fasting plasma glucose level was $\geq 126 \mathrm{mg} / \mathrm{dL}$, or the glycated hemoglobin A1c level was $\geq 6.5 \%$. The definition of dyslipidemia was a low density lipoprotein cholesterol level $\geq 140 \mathrm{mg} / \mathrm{dL}$, a high density lipoprotein cholesterol level $\leq 40 \mathrm{mg} / \mathrm{dL}$, a triglyceride level $\geq 150 \mathrm{mg} / \mathrm{dL}$, or use of lipid-lowering drugs. NIHSS scores were recorded at admission. The diagnosis of possible or probable CAA was based on the modified Boston criteria ${ }^{32}$.

\section{Detection of Cnm-Positive S. mutans}

Dental plaque specimens were collected and inoculated in Mitis-Salivarius medium with bacitracin (Sigma-Aldrich, St. Louis, MO, USA) and on $15 \%$ sucrose agar plates and anaerobically incubated at $37^{\circ} \mathrm{C}$ for 48 hours. S. mutans strains were identified and isolated on the basis of rough morphological features on the agar plates. The strains were then cultured in brain-heart infusion broth (Becton, Dickinson and Company, Franklin Lakes, NJ, USA) at $37^{\circ} \mathrm{C}$ for 24 hours. Bacterial genomic DNA was extracted from each strain. A polymerase chain reaction assay was performed using an $S$. mutans-specific primer set (forward, 5'-GGCACCACAACATTGGGAAGCTCAGTT-3'; reverse, 5'- GGAATGCCGATCAGTCAACAGGAT-3') according to a method described previously ${ }^{18}$. The presence or absence of the $\mathrm{cnm}$ gene was determined using primers designed to amplify the entire length of the $\mathrm{cnm}$ gene (forward, 5 '-

GACAAAGAAATGAAAGATGT-3'; reverse, 5'-GCAAAGACTCTTGTCCCTGC-3') ${ }^{18}$. Experiments were conducted by researchers who were blinded to the clinical information.

\section{Evaluation of CMBs}

In this study, CMBs were illustrated on T2*-weighted MRI. We evaluated the 'certain' brain microbleeds as CMBs according to the Brain Observer MicroBleed Scale ${ }^{5}$. The MRI parameters are summarized in the Supplementary Materials (see Supplemetary Table 3). We defined deep CMBs as hypointensities on T2*weighted MRI located in the deep gray matter of the basal ganglia or thalamus or the white matter of the corpus callosum or internal, external, or extreme capsule. Lobar CMBs were defined as those in the cortical gray or subcortical white matter. Infratentorial CMBs were defined as those in the brainstem or cerebellum. Strictly lobar CMBs were defined as CMBs restricted to a lobar region. We also classified deep and/or infratentorial and mixed $\mathrm{CMBs}^{33}$. Mixed CMBs included those located in both lobar and deep and/or infratentorial regions ${ }^{33}$. The term "all CMBs" encompasses CMBs in any brain region. The number 
of CMBs was independently determined by two trained neurologists blinded to the clinical data. In the case of disagreement, the opinion of a third neurologist was sought.

\section{Statistical Analyses}

Variables are presented as medians and interquartile ranges or numbers and percentages. The MannWhitney $U$ test was used to analyze continuous data, and the $\chi^{2}$ or Fisher exact test was used for categorical data. The numbers of all, deep, lobar, and infratentorial CMBs were stratified into six categories: $0,1,2-4,5-10,11-20$, and $\geq 21^{1,2}$. We examined the linear trend between Cnm-positive $S$. mutans and each $\mathrm{CMB}$ category using the Cochran-Armitage test. In addition, the association of Cnmpositive $S$. mutans with the number of CMBs was analyzed using quasi-Poisson regression models. The adjusted risk ratios and their $95 \%$ confidence intervals were estimated after adjustment for age, sex, systolic blood pressure, stroke type (ischemic stroke or ICH) and CAA (possible or probable CAA) ${ }^{34}$. A $P$ value $<0.05$ (two-tailed) was considered statistically significant. Statistical analyses were conducted using SPSS version 27 (IBM Corp., Armonk, NY, USA) and SAS version 9.4 (SAS Institute, Cary, NC, USA).

\section{Declarations}

\section{Acknowledgments}

We are indebted to Ms. Yuko Kiyama and Ms. Natsuki Hanada for technical assistance. We thank Lisa Kreiner, PhD, from Edanz (https://www.jp.edanz.com/ac) for editing a draft of this manuscript. This study was funded by a Grant-in-Aid from the Japan Society for the Promotion of Science Fellows (19J00106) and a Grant-in-Aid for Young Scientists to Dr Saito (21K16944), Grants-in-Aid for Challenging Exploratory Research to Dr Ihara (16K14573, 19K22610), funds from the Mitsui Sumitomo Insurance Welfare Foundation to Dr Ihara, and funds from the SENSHIN Medical Research Foundation to Dr Ihara.

\section{Author contributions statement}

Study conception: SS, RN, KN and MI. Data acquisition: SI, SS, SH, ST, Hajime I, and Hiroyuki I. Analysis and interpretation of data: SI, SS, Yumi Y, RN, MT and KN. Drafting the manuscript: SI, SS and MI.

Revising the manuscript critically for intellectual content: TT, YH, RPF, ROC, NK, Yusuke Y, HH, MK, and KT. Supervision of the study: SS, $\mathrm{HH}$ and MI.

\section{Additional information}

Koga reports honoraria from Bayer and Daiichi Sankyo; consultant fee from Ono pharmaceutical co., LTD; and research funds from Takeda, Daiichi Sankyo, Nippon Boehringer Ingelheim, Astellas and Shionogi. Toyoda reports lecture honoraria from Bayer, Bristol-Myers Squibb, Takeda, and Daiichi Sankyo. Ihara reports lecturer's fees from Daiichi Sankyo and Eisai, and grant support from Panasonic, GE Precision Healthcare LLC, Bristol-Myers Squibb, and Shimadzu Corporation.

\section{References}


1. Wilson, D. et al. Cerebral microbleeds and stroke risk after ischaemic stroke or transient ischaemic attack: a pooled analysis of individual patient data from cohort studies. Lancet Neuro/ 18, 653-665, doi:10.1016/S1474-4422(19)30197-8 (2019).

2. Charidimou, A. et al. Brain hemorrhage recurrence, small vessel disease type, and cerebral microbleeds: A meta-analysis. Neurology 89, 820-829, doi:10.1212/WNL.0000000000004259 (2017).

3. Akoudad, S. et al. Association of Cerebral Microbleeds With Cognitive Decline and Dementia. JAMA Neurol 73, 934-943, doi:10.1001/jamaneurol.2016.1017 (2016).

4. Petrault, M., Casolla, B., Ouk, T., Cordonnier, C. \& Berezowski, V. Cerebral microbleeds: Beyond the macroscope. Int J Stroke 14, 468-475, doi:10.1177/1747493019830594 (2019).

5. Cordonnier, C. et al. Improving interrater agreement about brain microbleeds: development of the Brain Observer MicroBleed Scale (BOMBS). Stroke 40, 94-99, doi:10.1161/STROKEAHA.108.526996 (2009).

6. van Veluw, S. J., Biessels, G. J., Klijn, C. J. \& Rozemuller, A. J. Heterogeneous histopathology of cortical microbleeds in cerebral amyloid angiopathy. Neurology 86, 867-871, doi:10.1212/WNL.0000000000002419 (2016).

7. Fazekas, F. et al. Histopathologic analysis of foci of signal loss on gradient-echo T2*-weighted MR images in patients with spontaneous intracerebral hemorrhage: evidence of microangiopathy-related microbleeds. AJNR Am J Neuroradiol 20, 637-642 (1999).

8. Jung, Y. H. et al. Strictly Lobar Microbleeds Reflect Amyloid Angiopathy Regardless of Cerebral and Cerebellar Compartments. Stroke 51, 3600-3607, doi:10.1161/STROKEAHA.119.028487 (2020).

9. Tonomura, S. et al. Intracerebral hemorrhage and deep microbleeds associated with cnm-positive Streptococcus mutans; a hospital cohort study. Sci Rep 6, 20074, doi:10.1038/srep20074 (2016).

10. Ihara, M. \& Yamamoto, Y. Emerging Evidence for Pathogenesis of Sporadic Cerebral Small Vessel Disease. Stroke 47, 554-560, doi:10.1161/STROKEAHA.115.009627 (2016).

11. Hosoki, S. et al. Oral Carriage of Streptococcus mutans Harboring the cnm Gene Relates to an Increased Incidence of Cerebral Microbleeds. Stroke 51, 3632-3639, doi:10.1161/STROKEAHA.120.029607 (2020).

12. Sato, Y. et al. Streptococcus mutans strains harboring collagen-binding adhesin. J Dent Res 83, 534539, doi:10.1177/154405910408300705 (2004).

13. Nakano, K. et al. The collagen-binding protein of Streptococcus mutans is involved in haemorrhagic stroke. Nat Commun 2, 485, doi:10.1038/ncomms1491 (2011).

14. Shiga, Y. et al. cnm-Positive Streptococcus mutans and diffusion-weighted imaging hyperintensities in acute intracerebral hemorrhage. Eur J Neurol 28, 1581-1589, doi:10.1111/ene.14725 (2021).

15. Watanabe, I. et al. Oral Cnm-positive Streptococcus Mutans Expressing Collagen Binding Activity is a Risk Factor for Cerebral Microbleeds and Cognitive Impairment. Sci Rep 6, 38561, doi:10.1038/srep38561 (2016). 
16. Nomura, R. et al. Potential involvement of Streptococcus mutans possessing collagen binding protein Cnm in infective endocarditis. Sci Rep 10, 19118, doi:10.1038/s41598-020-75933-6 (2020).

17. Okazaki, S. et al. Cerebral microbleeds predict impending intracranial hemorrhage in infective endocarditis. Cerebrovasc Dis 32, 483-488, doi:10.1159/000331475 (2011).

18. Nomura, R. et al. Molecular and clinical analyses of the gene encoding the collagen-binding adhesin of Streptococcus mutans. J Med Microbio/ 58, 469-475, doi:10.1099/jmm.0.007559-0 (2009).

19. Krasse, B., Edwardsson, S., Svensson, I. \& Trell, L. Implantation of caries-inducing streptococci in the human oral cavity. Arch Oral Biol 12, 231-236, doi:10.1016/0003-9969(67)90042-8 (1967).

20. Salonen, L., Allander, L., Bratthall, D. \& Hellden, L. Mutans streptococci, oral hygiene, and caries in an adult Swedish population. J Dent Res 69, 1469-1475, doi:10.1177/00220345900690080401 (1990).

21. Aviles-Reyes, A., Miller, J. H., Lemos, J. A. \& Abranches, J. Collagen-binding proteins of Streptococcus mutans and related streptococci. Mol Oral Microbio/ 32, 89-106, doi:10.1111/omi.12158 (2017).

22. Burns, E. M., Kruckeberg, T. W. \& Gaetano, P. K. Changes with age in cerebral capillary morphology. Neurobiol Aging 2, 283-291, doi:10.1016/0197-4580(81)90037-3 (1981).

23. Jandke, S. et al. The association between hypertensive arteriopathy and cerebral amyloid angiopathy in spontaneously hypertensive stroke-prone rats. Brain Pathol 28, 844-859, doi:10.1111/bpa.12629 (2018).

24. Soros, P., Whitehead, S., Spence, J. D. \& Hachinski, V. Antihypertensive treatment can prevent stroke and cognitive decline. Nat Rev Neuro/ 9, 174-178, doi:10.1038/nrneurol.2012.255 (2013).

25. Spence, J. D. The Importance of Blood Pressure Gradients in the Brain: Cerebral Small Vessel Disease. JAMA Neurol 76, 370-371, doi:10.1001/jamaneurol.2018.4627 (2019).

26. Blanco, P. J., Muller, L. O. \& Spence, J. D. Blood pressure gradients in cerebral arteries: a clue to pathogenesis of cerebral small vessel disease. Stroke Vasc Neuro/ 2, 108-117, doi:10.1136/svn2017-000087 (2017).

27. Lin, J. X. et al. White matter lesions and alteration of vascular cell composition in the brain of spontaneously hypertensive rats. Neuroreport 12, 1835-1839, doi:10.1097/00001756-20010703000015 (2001).

28. de Cavanagh, E. M. et al. Sympathetic predominance is associated with impaired endothelial progenitor cells and tunneling nanotubes in controlled-hypertensive patients. Am J Physiol Heart Circ Physio/ 307, H207-215, doi:10.1152/ajpheart.00955.2013 (2014).

29. Nanchahal, K., Ashton, W. D. \& Wood, D. A. Association between blood pressure, the treatment of hypertension, and cardiovascular risk factors in women. J Hypertens 18, 833-841, doi:10.1097/00004872-200018070-00003 (2000).

30. Dickinson, K. M., Clifton, P. M. \& Keogh, J. B. Endothelial function is impaired after a high-salt meal in healthy subjects. Am J Clin Nutr 93, 500-505, doi:10.3945/ajcn.110.006155 (2011).

31. Suvila, K. et al. Early Onset Hypertension Is Associated With Hypertensive End-Organ Damage Already by MidLife. Hypertension 74, 305-312, doi:10.1161/HYPERTENSIONAHA.119.13069 (2019). 
32. Greenberg, S. M. \& Charidimou, A. Diagnosis of Cerebral Amyloid Angiopathy: Evolution of the Boston Criteria. Stroke 49, 491-497, doi:10.1161/STROKEAHA.117.016990 (2018).

33. Yakushiji, Y. et al. Distribution of cerebral microbleeds in the East and West: Individual participant meta-analysis. Neurology 92, e1086-e1097, doi:10.1212/WNL.0000000000007039 (2019).

34. Vernooij, M. W. et al. Prevalence and risk factors of cerebral microbleeds: the Rotterdam Scan Study. Neurology 70, 1208-1214, doi:10.1212/01.wnl.0000307750.41970.d9 (2008).

\section{Figures}

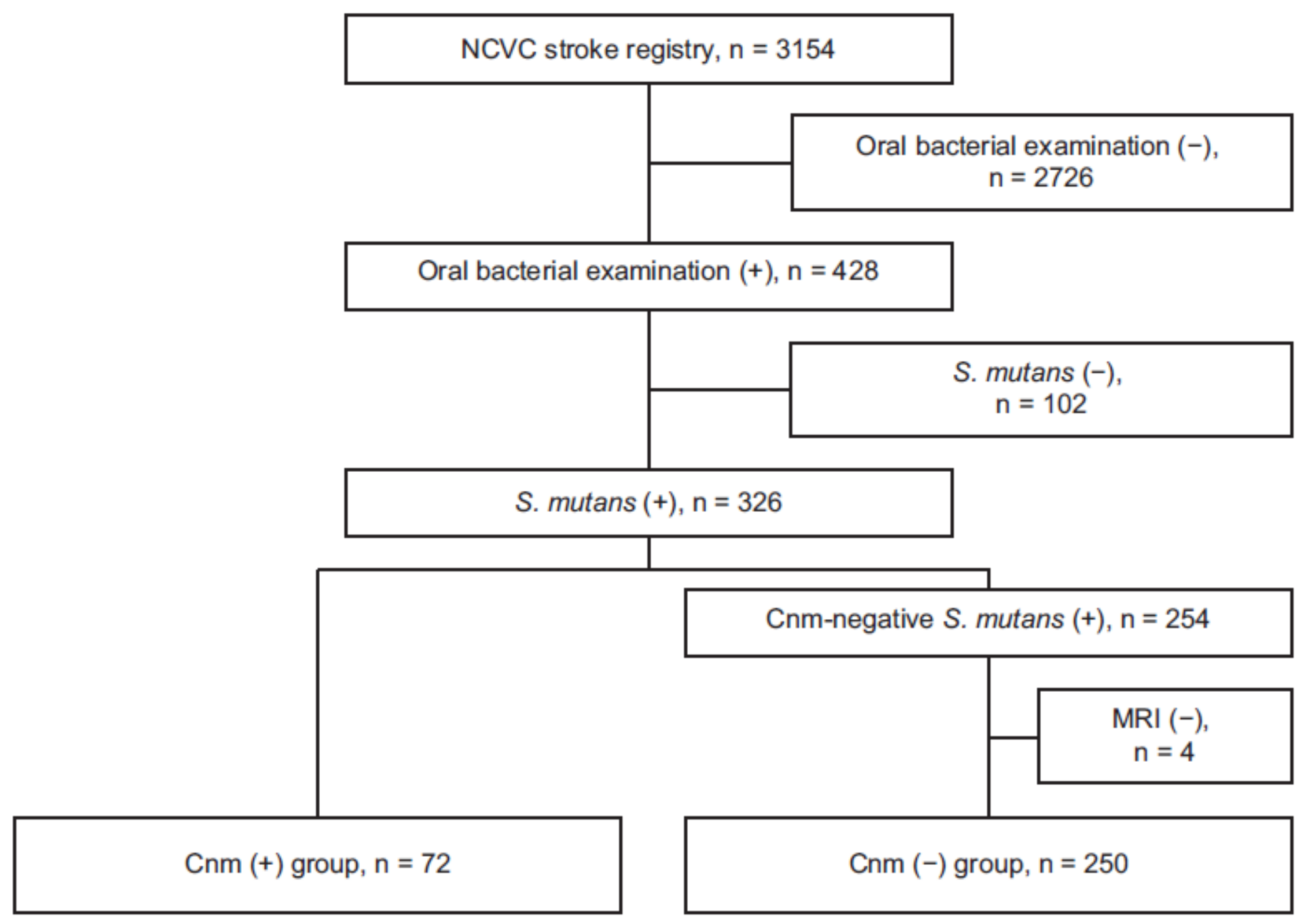

Figure 1

\section{Flow diagram of patient selection}

Patients with Cnm-positive S. mutans (Cnm [+] group) were compared with those with Cnm-negative $S$. mutans (Cnm [-] group). 




Figure 2

Representative images of cerebral microbleeds in a patient with Cnm-positive $S$. mutans

T2*-weighted magnetic resonance images of 59 -year-old man with $\mathrm{Cnm}$-positive $S$. mutans in his oral cavity showing multiple deep and lobar cerebral microbleeds.



Figure 3 


\section{Hypothetical model of Cnm-induced development of cerebral microbleeds}

Cerebral bleeding may occur at the level of arterioles and capillaries. Several factors including aging and hypertension result in endothelial damage and thickening of the basement membrane (BM). Cnm-positive $S$. mutans that enters the bloodstream after toothbrushing, flossing, or tooth extraction can attach to the exposed BM, where infiltration of neutrophils results in local inflammation, leading to cerebral microbleeds.

\section{Supplementary Files}

This is a list of supplementary files associated with this preprint. Click to download.

- supple12222SI.pdf 